\title{
Game theory and the frequency selective interference channel - A tutorial
}

\author{
Amir Leshem $^{1,2}$ and Ephraim Zehavi ${ }^{1}$
}

\begin{abstract}
This paper provides a tutorial overview of game theoretic techniques used for communication over frequency selective interference channels. We discuss both competitive and cooperative techniques.

Keywords: Game theory, competitive games, cooperative games, Nash Equilibrium, Nash bargaining solution, Generalized Nash games, Spectrum optimization, distributed coordination, interference channel, multiple access channel, iterative water-filling.
\end{abstract}

\section{COMMUNICATION OVER INTERFERENCE LIMITED CHANNELS}

The success of unlicensed broadband communication has led to very rapid deployment of communication networks that work independently of each other using a relatively narrow spectrum. For example the 802.11g standard is using the ISM band which has a total bandwidth of $60 \mathrm{MHz}$. This band is divided into 12 partially overlapping bands of $20 \mathrm{MHz}$. The success of these technologies might become their own limiting factor. The relatively small number of channels and the massive use of the technology in densely populated urban metropolitan areas will cause significant mutual interference. This is especially important for high quality real time distribution of multi-media content that is intolerant to errors as well as latency. Existing 802.11 (WiFi) networks have very limited means to coordinate spectrum with other interfering systems. It would be highly desirable to improve the interference environment by distributed spectral coordination between the different access points. Another scenario is that of centralized access points such as 802.16 (WiMax) where the resources are allocated centrally by a single base station. Similar situation is facing the advanced DSL systems such as ADSL2+ and VDSL. These systems are currently limited by crosstalk between the lines. As such the DSL environment is another example of highly frequency selective interference channel. While the need to operate over interference limited frequency selective channels is clear in many of the current and future communication technologies, the theoretical situation is much less satisfying. The capacity region of the interference channel is still open (see [1] for short overview) even for the fixed channel two user case. In recent years great advances in understanding the situation for flat channels under weak interference have been achieved. It can be

\footnotetext{
${ }^{1}$ School of Engineering, Bar-Ilan University, Ramat-Gan, 52900, Israel. ${ }^{2}$ Faculty of EEMCS, Delft University of Technology. This work was supported by Intel Corporation and by the Netherlands Foundation of Science and Technology. e-mail: leshema@eng.biu.ac.il .
} 
shown that in this case treating the interference as noise is almost optimal. On the other hand for the medium strength interference as is typical in the wireless environment, the simplest strategy is by using orthogonal signaling, e.g. TDMA/FDMA, for high spectral efficiency networks, or CDMA for very strong interference with low spectral efficiency per user. Moreover, sequential cancelation techniques that are required for the best known capacity region in the medium interference case [2] are only practical for small number of interferers. The interference channel is a conflict situation, and not every achievable rate pair (from informational point of view) is actually reasonable operating point for the users. This conflict is the reason to analyze the interference channel using game theoretical tools. Much work has been done on competitive game theory applied to frequency selective interference channel, with the early works of Yu et al. [3] and subsequent works of Scutari et al. (see [4] and the references therein). A particularly interesting topic is the use of generalized Nash games to the weak interference channel [5], and the algorithm in [6] which extends the FM-IWF to iterative pricing under fixed rate constraint.

The fact that competitive strategies can result in significant degradation due to the prisoner's dilemma has been called the price of anarchy [7]. In the interference channel case, a simple case has been analyzed by Laufer and Leshem [8], who characterized the cases of prisoner's dilemma in interference limited channels. To overcome the sub-optimality of the competitive approach we have two alternatives: Using repeated games or using cooperative game theory. Since most works on repeated games concentrated on flat fading channels, we will mainly concentrate on cooperative game theoretic approaches. One of the earliest solutions for cooperative games is the Nash bargaining solution [9]. Many papers in recent years were devoted to analyzing the Nash bargaining solution for the frequency flat interference channel in the SISO [10], [11], MISO [12], [13] and MIMO cases [14]. Interesting extensions for log-convex utility functions appeared in [15]. Another interesting application of the bargaining techniques discussed here is for multimedia distribution networks. Park and Van der Schaar [16] used both Nash bargaining and the generalized Kalai-Smorodinski solutions [17] for multimedia resource management. Another alternative cooperative model was explored in [18] where, the cooperation between rational wireless players was studied using coalitional game theory by allowing the receivers to cooperate using joint decoding.

In the context of frequency selective interference channels much less research has been done. Han et al. [19] in a pioneering work, studied the Nash Bargaining under FDM/TDM strategies and total power constraint. Unfortunately, the algorithms proposed were only sub-optimal. Iterative sub-optimal algorithms to achieve Nash bargaining solution for spectrum allocation under average power constraint have been applied in [20]. Only recently, we have managed to overcome the difficulties by imposing a total PSD mask constraint [21], in order to obtain computationally efficient solutions to the bargaining problem in the frequency selective SISO and MIMO cases under TDM/FDM strategies. Furthermore, it can be shown [22] that the PSD limited case can be used to derive a computationally efficient converging algorithm also in the total power constraint case. A very interesting problem is allowing the users to treat the interference as noise in some bands, while using orthogonal FDM/TDM strategies in others. This is a very challenging task, since the Nash bargaining solution involves a highly non-convex power allocation problem, instead of the simpler orthogonal signaling. 
As discussed before, the frequency selective interference channel is very interesting both from practical point of view and from information theoretic point of view. We can show that it has many interesting aspects from game theoretic point of view, and that various levels of interference admit different types of game theoretic technique. The purpose of this paper is to provide an overview of the various game theoretic techniques involved in the analysis and algorithms used for frequency selective interference channels. We demonstrate how game theory can be applied in specific scenarios and discuss signal processing aspects of the required game theoretic solutions. Our main goal is to discuss in a tutorial manner some new applications of game theory to frequency selective interference channels. First, we outline various interference channel scenarios with emphasis on frequency selective channels. Then we discuss the basic concepts of game theory required for analyzing these channels: Nash equilibrium, strategies, Generalized Nash games and Nash bargaining theory. Using these concepts we discuss the specific translations into working strategies for the communication models and discuss important signal processing aspects such as spectrum sensing and centralized and distributed strategies. To wrap up the discussion with real life applications, end up with two case studies: DSL and WiMax where we demonstrate the gains of the techniques on real channels. We end up with conclusions and future research directions.

\section{INTRODUCTION TO INTERFERENCE CHANNELS}

Computing the capacity region of the interference channel is an open problem in information theory [23]. A good overview of the results until 1985 is given by van der Meulen [1] and the references therein. The capacity region of general interference channel is not known yet. However, in the last forty five years of research some progress has been made. The best known achievable region for the general interference channel is due to Han and Kobayashi [2]. The computation of the Han and Kobayashi formula for a general discrete memoryless channel is in general too complex. Recently large advance in obtaining upper bounds on the rate region have been obtained especially for the case of weak interference.

A 2x2 Gaussian interference channel in standard form (after suitable normalization) is given by:

$$
\mathbf{x}=\mathbf{H} \mathbf{s}+\mathbf{n}, \quad \mathbf{H}=\left[\begin{array}{cc}
1 & \alpha_{1} \\
\alpha_{2} & 1
\end{array}\right]
$$

where, $\mathbf{s}=\left[s_{1}, s_{2}\right]^{T}$, and $\mathbf{x}=\left[x_{1}, x_{2}\right]^{T}$ are sampled values of the input and output signals, respectively. The noise vector $\mathbf{n}$ represents the additive Gaussian noises with zero mean and unit variance. The powers of the input signals are constrained to be less than $P_{1}, P_{2}$, respectively. The off-diagonal elements of $\mathbf{H}, \alpha_{1}, \alpha_{2}$ represent the degree of interference present. The major difference between the interference channel and the multiple access channel is that both encoding and decoding of each channel are performed separately and independently, with no information sharing between receivers.

The capacity region of the Gaussian interference channel with very strong interference (i.e., $\alpha_{1} \geq 1+P_{1}$, $\left.\alpha_{2} \geq 1+P_{2}\right)$ is given by [24]

$$
R_{i} \leq \log _{2}\left(1+P_{i}\right), \quad i=1,2
$$


This surprising result shows that very strong interference dose not reduce the capacity of the users. A Gaussian interference channel is said to have strong interference if $\min \left\{\alpha_{1}, \alpha_{2}\right\}>1$. Sato [25] derived an achievable capacity region (inner bound) of Gaussian interference channel as intersection of two multiple access Gaussian capacity regions embedded in the interference channel. The achievable region is the intersection of the rate pair of the rectangular region of the very strong interference (2) and the following region:

$$
R_{1}+R_{2} \leq \log _{2}\left(\min \left\{1+P_{1}+\alpha P_{2}, 1+P_{2}+\beta P_{1}\right\}\right) .
$$

While the two user flat interference channel is a well studied (although not solved) problem, much less is known in the frequency selective case. An $N \times N$ frequency selective Gaussian interference channel is given by:

$$
\begin{aligned}
& \mathbf{x}_{k}=\mathbf{H}_{k} \mathbf{s}_{k}+\mathbf{n}_{k} \quad k=1, \ldots, K \\
& \mathbf{H}_{k}=\left[\begin{array}{ccc}
h_{11}(k) & \ldots & h_{1 N}(k) \\
\vdots & \ddots & \vdots \\
h_{N 1}(k) & \ldots & h_{N N}(k)
\end{array}\right] .
\end{aligned}
$$

where, $\mathbf{s}_{k}$, and $\mathbf{x}_{k}$ are sampled values of the input and output signal vectors at frequency $k$, respectively. The noise vector $\mathbf{n}_{k}$ represents an additive white Gaussian noise with zero mean and unit variance. The power spectral density (PSD) of the input signals are constrained to be less than $p_{1}(k), p_{2}(k)$ respectively. Alternatively, only a total power constraint is given. The off-diagonal elements of $\mathbf{H}_{k}$, represent the degree of interference present at frequency $k$. The main difference between interference channel and a multiple access channel (MAC) is that in the interference channel, each component of $s_{k}$ is coded independently, and each receiver has access to a single element of $\mathbf{x}_{k}$. Therefore, iterative decoding schemes are much more limited, and typically impractical for large number of users.

To overcome this problem there are two simple strategies. When the interference is sufficiently weak, the common wisdom is to treat the interference as noise, and code at a rate corresponding to the total noise. When the interference is stronger, i.e, Signal to Interference Ratio (SIR) is significantly lower than Signal to additive Noise Ratio (SNR), treating the interference as noise can be highly inefficient. One of the simplest ways to deal with medium to strong interference channels is through orthogonal signaling. Two extremely simple orthogonal schemes are using FDM or TDM strategies. These techniques allow a single user detection (which will be assumed throughout this paper) without the need to complicated multi-user detection. The loss of these techniques compared to techniques requiring joint decoding has been thoroughly studied, e.g., [24] in the constant channel case, showing degradation compared to the techniques requiring joint or sequential decoding. However, the widespread use of FDMA/TDMA as well as collision avoidance medium access control (CSMA) techniques, make the analysis of these techniques very important from practical point of view as well. For frequency selective channels (also known as ISI channels) we can combine both strategies by allowing time varying allocation of the frequency bands to the different users as shown in figure $1(\mathrm{~b})$ 


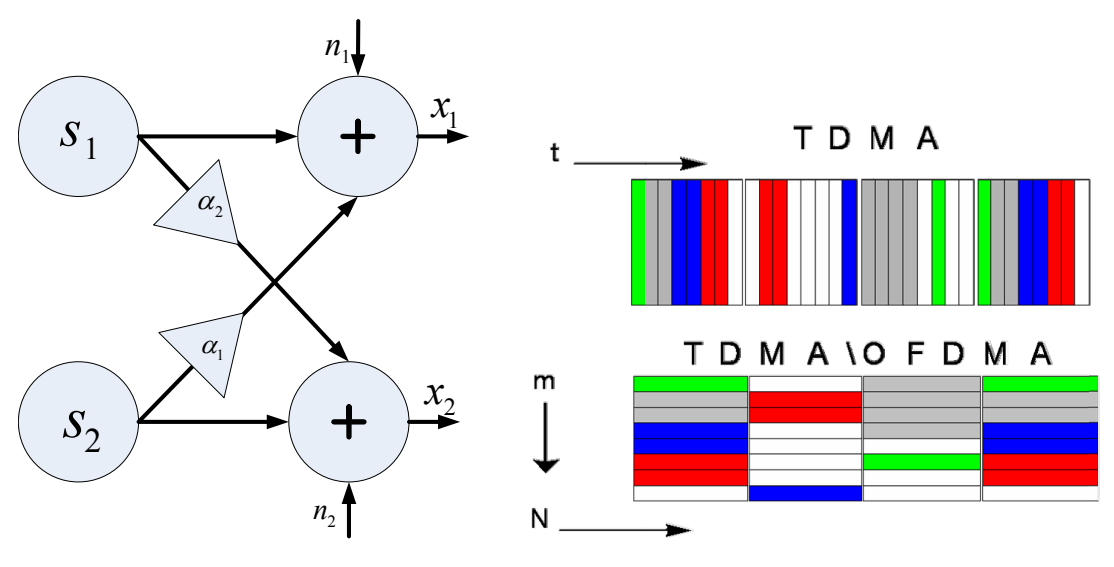

(a) Interference channel

(b) TDMA and joint TDMA/OFDMA

Fig. 1. (a) Standard form interference channel. (b) TDMA and joint TDMA/OFDMA

In this paper we limit ourselves to joint FDM and TDM scheme where an assignment of disjoint portions of the frequency band to the several transmitters is made at each time instance. This technique is widely used in practice because simple filtering can be used at the receivers to eliminate interference. All of these schemes as to operate under physical and regulation constraint like Average power constraint or/and PSD mask constraint.

\section{BASIC CONCEPTS OF COOPERATIVE AND COMPETITIVE GAME THEORY}

In this section we review the basic concepts of game theory in an abstract setting. Our focus is on concepts that have been found to be relevant to the frequency selective interference channel. We begin with competitive game theory and then continue to describe the cooperative solutions. The reader is referred to the excellent books of [26], [27] and [28] for more details and for proofs of the main results mentioned here.

\section{A. Static competitive games and the Nash equilibrium}

An static $N$ player game in strategic form is a three tuple $(N, A, \mathbf{u})$ composed of a set of players $\{1, \ldots, N\}$, a set of possible combinations of actions of each player denoted by $A=\prod_{n=1}^{N} A_{n}$, where $A_{n}$ is the set of actions for the $n$ 'th player and a vector utility function $\mathbf{u}=\left[u_{1}, \ldots, u_{N}\right]$, where $u_{n}\left(a_{1}, \ldots, a_{N}\right)$ : $\prod_{n=1}^{N} A_{n} \rightarrow \mathcal{R}$ is the utility of the $n$ 'th player when strategy vector $\mathbf{a}=\left(a_{1}, \ldots, a_{N}\right)$ has been played. The interpretation of $u_{n}$ is that player $n$ receives a payoff of $u_{n}\left(a_{1}, \ldots, a_{N}\right)$ when the players have chosen actions $a_{1}, \ldots, a_{N}$. The game is finite when for all $n, A_{n}$ is a finite set.

A special type of competitive games are the constant sum games. A game is constant sum, if for all action vectors $\mathbf{a}, \sum_{n=1}^{N} u_{n}(\mathbf{a})=c$ for some constant $c$. When the game is constant sum we can subtract $c / N$ from each utility and obtain a zero sum game that has the same properties as the original game. A 
two players zero sum game is strictly competitive since anything gained by one player leads to a loss to the other player.

An important notion of solution relevant to games is that of a Nash equilibrium.

Definition 3.1: A vector of actions $\mathbf{a}=\left(\mathbf{a}_{1}, \ldots, \mathbf{a}_{N}\right) \in A$ is a Nash equilibrium in pure strategies if and only if for each player $1 \leq n \leq N$ and for every $\mathbf{a}^{\prime}=\left(a_{1}^{\prime}, \ldots, a_{N}^{\prime}\right)$ such that $a_{i}^{\prime}=\mathbf{a}_{i}$ for all $i \neq n$ and $a_{n}^{\prime} \neq a_{n}$ we have $u_{n}\left(\mathbf{a}^{\prime}\right)<u_{n}(\mathbf{a})$, i.e., each player can only loose by deviating by itself from the equilibrium.

The Nash equilibrium in pure strategies does not always exist as the following example shows:

Example I - A game with no pure strategy Nash equilibrium: Consider the two players game defined by the following: $A_{i}=\{0,1\} \cdot u_{i}\left(a_{1}, a_{2}\right)=a_{1} \oplus a_{2} \oplus(i-1)$, i.e., the first player payoff is 1 when actions are identical and 0 otherwise, while the second player's payoff is 1 when the actions are different and 0 otherwise. Clearly, this game also known as matching pennies has no Nash equilibrium in pure strategies, since always one of the players can improve his situation by changing his choice.

Even when it exists, the Nash equilibrium in pure strategies is not necessarily unique, as the following example shows:

Example II - A communication game with infinitely many pure strategy NE. Assume that two users are sharing an AWGN multiple access channel (i.e., the accss point can perform joint decoding of the users)

$$
y=x_{1}+x_{2}+z
$$

where $z \sim N\left(0, \sigma^{2}\right)$ is a Gaussian noise random variable. Each user has power $P$. It is well known [23] that the rate region of this multiple access channel is given by a pentagon defined by:

$$
\begin{aligned}
& R_{1} \leq \frac{1}{2} \log \left(1+\frac{P}{\sigma^{2}}\right)=C^{\max } \\
& R_{2} \leq \frac{1}{2} \log \left(1+\frac{P}{\sigma^{2}}\right)=C^{\max } \\
& R_{1}+R_{2} \leq \frac{1}{2} \log \left(1+\frac{2 P}{\sigma^{2}}\right)=C_{1,2} .
\end{aligned}
$$

The corners $A, B$ (see figure 2(a) of the pentagon are $A=\left(C^{\max }, C^{\min }\right)$ and $B=\left(C^{\text {min }}, C^{\text {max }}\right)$, where $C^{\text {min }}=\frac{1}{2} \log \left(1+\frac{P}{P+\sigma^{2}}\right)$, is the rate achievable by assuming that the other user's signal is interference. Note that any point on the line connecting the points $A, B$ is achieved by time sharing between these two points. Each player $n=1,2$ can choose a strategy $0 \leq \alpha_{n} \leq 1$ that is the time sharing ratio between coding at its rate at point $A$ or $B$. The payoff in this game is given by

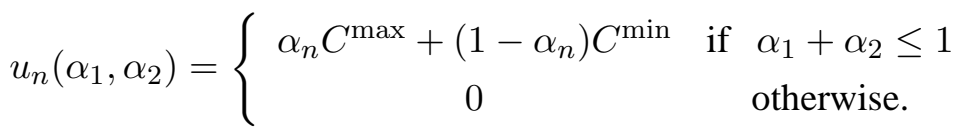

The reason that the utility is 0 when $\alpha_{1}+\alpha_{2}>1$ is that no reliable communication is possible, since the rate pair achieved is outside the rate region. In this game any valid strategy point such that $\alpha_{1}+\alpha_{2}=1$ is a Nash equilibrium. If user $n$ reduces its $\alpha_{n}$ obviously its rate is lower since he transmits larger fraction of the time at the lower rate. If on the other hand he increases $\alpha_{n}$ then $\alpha_{1}+\alpha_{2}>1$ and both players achieve 0 . Hence the AWGN MAC game has infinitely many Nash equilibrium points. Similar game has 
been used in [29] where the fact that infinitely many NE points exist is shown. It is interesting to note that a similar MAC game for the fading channel has a unique Nash equilibrium point [30].

To better understand this game, we might look at the best response dynamics. Best response action is the attempt of a player to maximize its utility against a given strategy vector. It is a well established mean of distributively achieving the Nash Equilibrium. In the context of information theory, this strategy has been termed Iterative Water-Filling (IWF) [3]. If in the multiple access game the players use the best response simultaneously, the first step would be to transmit at $C^{\max }$. Each player then receives 0 utility and in the next step reduces its rate to $C^{\mathrm{min}}$, and vice versa. The iteration never converges and the utility of each player is given by $\frac{1}{2} C^{\mathrm{min}}$, worse than transmitting constantly at $C^{\mathrm{min}}$. Interestingly in this case, the sequential best response leads to one of the points $A, B$, which are the (non axis) corners of the rate region. The moral of this example is that using the best response strategy should be done carefully even in multiple access scenario's such as in [31].

\section{B. Pure and mixed strategies}

To overcome the first problem of no equilibrium in pure strategies, the notion of mixed strategy has been proposed.

Definition 3.2: A mixed strategy $\pi_{n}$ for player $n$ is a probability distribution over $A_{n}$.

The interpretation of the mixed strategies is that player $n$ chooses his action randomly from $A_{n}$ according to the distribution $\pi_{n}$. The payoff of player $n$ in a game where the mixed strategies $\pi_{1}, \ldots, \pi_{N}$ are played by the players is the expected value of the utility

$$
u_{n}\left(\pi_{1}, \ldots, \pi_{N}\right)=E_{\pi_{1} \times \ldots \times \pi_{N}}\left[u_{n}\left(x_{1}, \ldots, x_{N}\right)\right] .
$$

Example III: Mixed strategies in random access game over multiple access channel To demonstrate the notion of mixed strategy, we now extend the multiple access game, into a random multiple access game, where the players can choose with probability $p_{n}$ of working at rate $C^{\min }$ and $1-p_{n}$ working at $C^{\max }$. This replaces the synchronized TDMA strategy in the previous game, with slotted random access protocol. This formulation, allows for two pure strategies corresponding to the corner points $A, B$ and the mixed strategies amount to randomly choosing between these points. This game is a special case of the chicken dilemma (a termed proposed by B. Russel, [32]), since for each user it is better to chicken out, then to obtain zero rate, when both players choose the tough strategies. Obviously from the previous discussion, the points $\left(C^{\max }, C^{\min }\right)$ and $\left(C^{\min }, C^{\max }\right)$ are Nash equilibria. Simple computation shows that there is a unique Nash equilibrium in mixed strategy corresponding to $p_{1}=p_{2}=C^{\min } / C^{\max }$. Interestingly the rates achieved by this random access (mixed strategy) approach is exactly $\left(C^{\min }, C^{\text {min }}\right)$, i.e., the price paid for random access is that both players achieve their minimal rate, so simple $p$-persistent random access provides no gain for the multiple access channel. Following Papadimitriou [7] we can call this the price of random access. 
TABLE I

PAYOFFS IN THE MULTICHANNEL RANDOM ACCESS GAME

\begin{tabular}{c|c|c|}
$\mathrm{I} \backslash \mathrm{II}$ & 0 & 1 \\
\hline 0 & $\left(C^{\min }, C^{\min }\right)$ & $\left(C^{\min }, C^{\max }\right)$ \\
\hline 1 & $\left(C^{\max }, C^{\min }\right)$ & $(0,0)$ \\
\hline
\end{tabular}

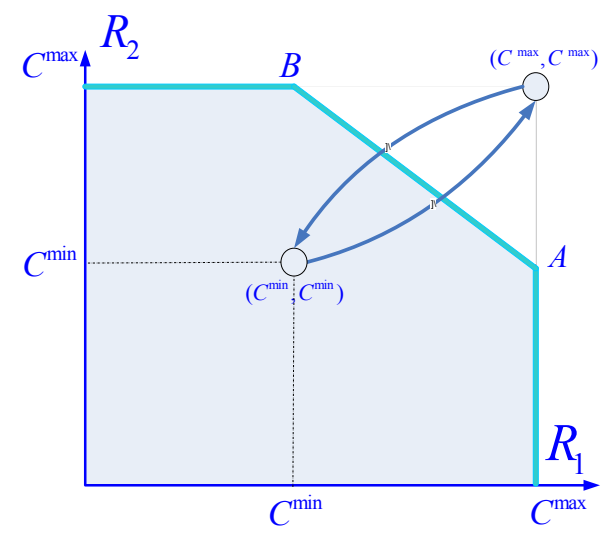

(a) Multiple Access game

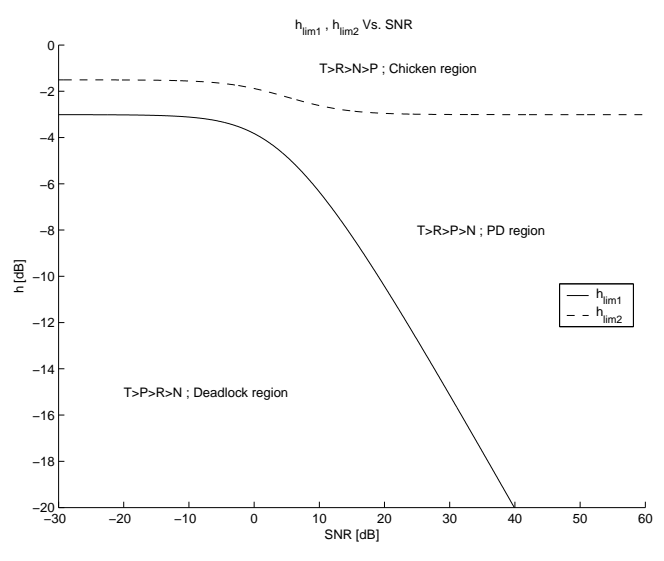

(b) Prisoner's dilemma regions

Fig. 2. (a) Multiple Access Game with infinitely many pure strategy equilibria. Note that the parallel best response dynamics leads to unstable dynamics while the serial best response leads to one of two extremal NE points. (b) Graph of $h_{\text {lim } 1}, h_{\text {lim } 2}$ Vs. SNR, The solid line corresponds to $h_{\lim 1}$ and the dashed line corresponds to $h_{\lim 2}$ [8].

\section{C. convex games}

An important special type of games which is important for the spectrum management problem is that of convex competitive games.

Definition 3.3: An $N$ player game $(N, A, U)$ is convex if each $A_{n}$ is compact and convex and each $u_{n}\left(x_{1}, \ldots, x_{n}\right)$ is a convex function of $x_{n}$ for every choice of $\left\{x_{j}: j \neq n\right\}$.

Convex games always have Nash equilibrium [33]. A simple proof can be found in [27]. Convex competitive games are especially important in the context of spectrum management, since the basic Gaussian interference game forms a convex game.

\section{The prisoner's dilemma}

The prisoner's dilemma game is the major problem of the competitive approach. It was first described by Flood and Dresher in 1950 almost immediately after the concept of Nash equilibrium was published [9]. For an overview of the prisoner's dilemma and its history see the excellent book of Poundstone [32] or in this journal [34]. It turns out that this game has a unique Nash equilibrium which is the stable point of the game. Moreover this outcome is suboptimal to all player. The emergence of the prisoner's dilemma 
in simple symmetric interference channels has been discussed in [8]. In [10], [21] characterization of cases where cooperative solutions are better for general interference channels have been demonstrated. We briefly describe a simple case where the prisoner's dilemma occurs [8]. We assume a simplified two players game. The game is played over two frequency bands each with symmetric interference channel. The channel matrices of this channel are $\mathbf{H}(1)=\mathbf{H}(2)=\mathbf{H}$ where

$$
\left|h_{12}(1)\right|^{2}=\left|h_{21}(1)\right|^{2}=\left|h_{12}(2)\right|^{2}=\left|h_{21}(2)\right|^{2}=h
$$

and $h_{i i}(k)=1$. We limit the discussion to $0 \leq h<1$. In our symmetric game both users have the same power constraint $P$ and the power is allocated by $p_{1}(1)=(1-\alpha) P, p_{1}(2)=\alpha P, p_{2}(1)=\beta P, p_{2}(2)=$ $(1-\beta) P$. We assume that the decoder treats the interference as noise and cannot decode it. The utility for user I given power allocation parameters $\alpha, \beta$ is given by its achievable rate

$$
C^{1}=\frac{1}{2} \log _{2}\left(1+\frac{(1-\alpha)}{S N R^{-1}+\beta \cdot h}\right)+\frac{1}{2} \log _{2}\left(1+\frac{\alpha}{S N R^{-1}+(1-\beta) \cdot h}\right)
$$

and similarly for user II, we replace $\alpha, \beta$. The set of strategies in this simplified game is $\{\alpha, \beta: 0 \leq \alpha, \beta \leq 1\}$. There are four alternatives outcomes:

- Both users select FDM resulting in $\alpha=\beta=0$. This is the reward (R) for cooperation.

- Player I selects FDM while user II selects competitive approach (IWF) resulting in $\alpha=0, \beta=$ $(1-h) / 2$ is the result of waterfilling by user II when $\alpha=0)$. This is when player I is naive (N) and the temptation for player II (T)

- Player I selects IWF while user II selects FDM resulting in $\alpha=(1-h) / 2, \beta=0$. This is the temptation for player I (T) and player II is the naive $(\mathrm{N})$.

- Both players select IWF resulting in $\alpha=\beta=\frac{1}{2}$. This is the penalty for not cooperating (P).

Table $\Pi$ describes the payoffs of users I at four different levels of mutual cooperation (The payoffs of user II are the same with the inversion of the cooperative/competetive roles). A prisoner's dilemma situation

TABLE II

USER I PAYOFFS AT DIFFERENT LEVELS OF MUTUAL COOPERATION

\begin{tabular}{|c|c|c|}
\hline & $\begin{array}{c}\text { user II is fully cooperative } \\
(\beta=0)\end{array}$ & $\begin{array}{c}\text { user II is fully competing } \\
\left(\beta=\frac{(2 \alpha-1) h+1}{2}\right)\end{array}$ \\
\hline $\begin{array}{c}\text { user I is fully cooperative } \\
(\alpha=0)\end{array}$ & $\frac{1}{2} \log _{2}\left(1+\frac{1}{S N R^{-1}}\right)$ & $\frac{1}{2} \log _{2}\left(1+\frac{1}{S N R^{-1}+\frac{(1-h)}{2}}\right)$ \\
\hline $\begin{array}{c}\text { user I is fully competing } \\
\left(\alpha=\frac{(2 \beta-1) h+1}{2}\right)\end{array}$ & $\frac{1}{2} \log _{2}\left(1+\frac{\frac{1+h}{2}}{S N R^{-1}}\right)+\frac{1}{2} \log _{2}\left(1+\frac{\frac{1-h}{2}}{S N R^{-1}+h}\right)$ & $\log _{2}\left(1+\frac{\frac{1}{2}}{S N R^{-1}+\frac{1}{2} h}\right)$ \\
\hline
\end{tabular}

is defined by the following payoff relations for both players $-T>R>P>N$. It is easy to show that the Nash equilibrium point in this case is that both players will defect $(P)$. This is caused by the fact that given the other user act the best response will be to defect (since $T>R$ and $P>N$ ). Obviously a better strategy (which makes this game a dilemma) is mutual cooperation (since $R>P$ ). We can now analyze 
this simple game. It turns out that there are two functions, $h_{\lim _{1}}(S N R), h_{\lim _{2}}(S N R)$ as described in Figure 2(b) and only three possible situations [8]:

- (A) $T>P>R>N$, for $h<h_{\lim _{1}}$.

- (B) $T>R>P>N$, for $h_{\lim _{1}}<h<h_{\lim _{2}}$.

- (C) $T>R>N>P$, for $h_{\lim _{2}}<h$.

The payoff relations in (A) corresponds to a game called "Deadlock". In this game there is no dilemma, since no matter what the other player does, it is better to defect $(T>R$ and $P>N$ ), so the Nash equilibrium point is $P$. Since $P>R$ thus there is no reason to cooperate. The maximum sum rate is also $P$ because $2 \cdot R>T+N$ and $P>R$. The payoff relations in (B) correspond to the prisoner's dilemma situation. While the Nash equilibrium point is $P$, each user's maximum payoff is achieved by $R$. In this region the FDM strategy will achieve the individual maximum rate. The last payoff relations (C) corresponds to a game called "Chicken". This game has two Nash equilibrium points, $T$ and $N$. This is caused by the fact that for each of the other player's strategies the opposite response is preferred (if the other cooperates it is better to defect since $T>R$, while if the other defects it is better to cooperate since $N>P$ ). The maximum rate sum point is still at $R$ (since $R>P$ and $2 \cdot R>T+N$ ) thus, again FDM will achieve the maximum rate sum while IWF will not.

\section{E. Generalized Nash games}

Games in strategic forms are very important part of game theory, and have many applications. However, in some cases the notion of a game does not capture all the complications involved in the interaction between the players. Arrow and Debreu [35] defined the concept of a generalized Nash game and generalized Nash equilibrium. In strategic form games, each player has a set of strategies that is independent from the actions of the other players. However, in reality sometimes the actions of the players are constrained by the actions of the other players. The generalized Nash game or abstract economy concept, captures exactly this dependence.

Definition 3.4: A generalized Nash game with $N$ players, is defined as follows: For each player $n$ we have a set of possible actions $X_{n}$ and a set function $K_{n}: \prod_{m \neq n} X_{m} \rightarrow P\left(X_{n}\right)$ where $P(X)$ is the power set of $X$, i.e, $K_{n}\left(\left\langle x_{m}: m \neq n\right\rangle\right) \subseteq X_{n}$ defines a subset of possible actions for player $n$ when other players play $\left\langle x_{m}: m \neq n\right\rangle . u_{n}(\mathbf{x})$ is a utility function defined on all tuples $\left(x_{1}, \ldots, x_{N}\right)$ such that $x_{n} \in K_{n}\left(x_{m}: m \neq n\right)$.

Similarly to the definition of a Nash equilibrium, we can define a generalized Nash equilibrium:

Definition 3.5: A generalized Nash equilibrium is a point $\mathbf{x}=\left(x_{1}, \ldots, x_{N}\right)$ such that for all $n x_{n} \in$ $K_{n}\left(\left\langle x_{m}: m \neq n\right\rangle\right)$, and for all $\mathbf{y}=\left(y_{1}, \ldots, y_{n}\right)$ such that $y_{n} \in K_{n}\left(\left\langle x_{m}: m \neq n\right\rangle\right)$ and $y_{m}=x_{m}$ for $m \neq n u_{n}(\mathbf{x}) \geq u_{n}(\mathbf{y})$.

Arrow and Debreu [35] proved the existence of a generalized Nash equilibrium under very limiting conditions. Their result was generalized, and currently the best result is by Ichiishi [36]. This result can be used to analyze certain fixed rate and margin versions of the iterative water-filling algorithm [5] as will be shown in the next section. 


\section{F. Nash bargaining theory}

The "Prisoner Dilema" highlights the drawbacks of competition, due to mutual mistrust of players. We therefore ask ourselves when would a cooperative strategy be preferable to a competitive strategy. It is apparent that the essential condition for cooperation is that it should generate a surplus, i.e. an extra gain which can be divided between the parties. Bargaining is essentially the process of distributing the surplus. Thus, bargaining is foremost, a process in which parties seek to reconcile contradictory interests and values. The main question is if all players commit to following the rules, what reasonable outcome is acceptable by all parties. Therefore, the players have to agree on a bargaining mechanism which they will not abandon during the negotiations. The bargaining results may be affected by several factors like the power of each party, the amount of information available to each of the players, the delay response of the players, etc. Nash [9], [37] introduced an axiomatic approach that is based on properties that a valid outcome of the bargaining should satisfy. This approach proved to be very useful since it succeeded in choosing a unique solution through a small number of simple conditions (axioms), thus saving the need to perform the complicated bargaining process, once all parties accept these conditions.

We now define the bargaining problem. An n-player bargaining problem is described as a pair $\langle S, d\rangle$, where $S$ is a convex set in $n$-dimensional Euclidean space, consisting of all feasible sets of $n$-players utilities that the players can receive when cooperating. $d$ is an element of $S$, called the disagreement point, representing the outcome if the players fail to reach an agreement. $d$ can also can be viewed as the utilities resulting from non-cooperative strategy (competition) between the players, which is a Nash Equilibrium of a competitive game. The assumption that $S$ is a convex set, is a reasonable assumption if both players select cooperative strategies, since, the players can use alternating or mixed strategies to achieve convex combinations of pure bargaining outcomes. Given a bargaining problem we say that the vector $\mathbf{u} \in S$ is Pareto optimal if for all $\mathbf{w} \in S$ if $\mathbf{w} \geq \mathbf{u}$ (coordinate wise) then $\mathbf{w}=\mathbf{u}$. A solution to the bargaining problem is a function $F$ defined on all bargaining problems such that $F(\langle S, d\rangle) \in S$. Nash's axiomatic approach is based on the following four axioms that the solution function $F$ should satisfy:

Linearity (LIN). Assume that we consider the bargaining problem $\left\langle S^{\prime}, d^{\prime}\right\rangle$ obtained from the problem $\langle S, d\rangle$ by transformations: $s_{n}^{\prime}=\alpha_{n} s_{n}+\beta_{n}, \quad n=1, \ldots, N . d_{n}^{\prime}=\alpha_{n} d_{n}+\beta_{n}$. Then the solution satisfies $F_{i}\left(\left\langle S^{\prime}, d^{\prime}\right\rangle\right)=\alpha_{n} F_{n}(\langle S, d\rangle)+\beta_{n}$, for all $n=1, \ldots, N$.

Symmetry $(S Y M)$. If two players $m<n$ are identical in the sense that $S$ is symmetric with respect to changing the $m$ 'th and the $n$ 'th coordinates, then $F_{m}(\langle S, d\rangle)=F_{n}(\langle S, d\rangle)$. Equivalently, players which have identical bargaining preferences, get the same outcome at the end of the bargaining process.

Pareto optimality $(P A R)$. If $\mathbf{s}$ is the outcome of the bargaining then no other state $\mathbf{t}$ exists such that $\mathbf{s}<\mathbf{t}$ (coordinate wise).

Independence of irrelevant alternatives (IIA). If $S \subseteq T$ and if $F(\langle T, d\rangle)=\left(u_{1}^{*}, u_{2}^{*}\right) \in S$, then $F(\langle S, d\rangle)=\left(u_{1}^{*}, u_{2}^{*}\right)$. 
Surprisingly these simply axioms fully characterize a bargaining solution titled Nash's Bargaining solution. Nash's theorem may be stated as follows [9].

Based on this axioms and definitions we now can state Nash's theorem.

Theorem 3.1: Assume that for all $S$ is compact and convex, then there is a unique bargaining solution $F(\langle S, d\rangle)$, satisfying the axioms INV, SYM, IIA, and PAR, which is given by

$$
F(\langle S, d\rangle)=\left(s_{1}, . ., s_{N}\right)=\arg \max _{\left(d_{1}, . ., d_{N}\right) \leq\left(s_{1}, . ., s_{N}\right) \in S} \prod_{n=1}^{N}\left(s_{n}-d_{n}\right) .
$$

Before continuing the study of the bargaining solution, we add a cautionary word. While, Nash's axioms are mathematically appealing, they may not be acceptable in some scenarios. Indeed various alternatives to these axioms have been proposed that lead to other solution concepts. Extensive survey of these solutions can be found in [17]. In the communication context, the axioms proposed by Boche at. el. [11] lead to a generalized NBS solution. More results of generalized bargaining for frequency selective channels will be discussed in [22]. To demonstrate the use of the Nash bargaining solution to interference channels, we begin with a simple example for flat channels.

Example III: Consider two players communicating over a 2x2 memoryless Gaussian interference channel with bandwidth $W=1$, as described in (1) Assume for simplicity that $P_{1}=P_{2}=P$. We assume that no receiver can perform joint decoding, and the utility of player $n, U_{n}$, is given by the achievable rate $R_{n}$. Similarly to the prisoner's dilemma example, if the players choose to compete then the competitive strategies in the interference game are given by flat power allocation and the resulting rates are given by $R_{n C}=1 / 2 \log _{2}\left(1+\frac{P}{1+\alpha_{n}^{2} P}\right)$. Since the rates $R_{n C}$ are achieved by competitive strategy, player $n$ would cooperate only if he will obtain a rate higher than $R_{n C}$. The game theoretic rate region is defined by pair rates higher than the competitive rates $R_{n C}$ (see figure 3(b)]. Assume that the players agree on using only FDM cooperative strategies. i.e. player $n$ uses a fraction of $0 \leq \rho_{n} \leq 1$ of the band. If we consider only Pareto optimal strategy vectors, then obviously $\rho_{2}=1-\rho_{1}$. The rates

obtained by the two users are given by $R_{n}\left(\rho_{n}\right)=\frac{\rho_{n}}{2} \log _{2}\left(1+\frac{P}{\rho_{n}}\right)$. The two users will benefit from FDM type of cooperation as long as

$$
R_{n C} \leq R_{n}\left(\rho_{1}\right), \quad n=1,2 .
$$

The FDM Nash bargaining solution is given by solving the problem

$$
\max _{\rho} F(\rho)=\max _{\rho}\left(R_{1}(\rho)-R_{1 C}\right)\left(R_{2}(\rho)-R_{2 C}\right) .
$$

The cooperative solution for this flat channel model was derived in [10], [21].

\section{APPLICATION OF GAME THEORY TO FREQUENCY SELECTIVE INTERFERENCE CHANNELS}

In this section we apply the ideas presented in previous sections to analyze the frequency selective interference game. We provide examples for both competitive and cooperative game theoretic concepts. 


\section{A. Waterfilling based solutions and the Nash equilibrium}

To analyze the competitive approach to frequency selective interference channels, we first define the discrete-frequency Gaussian interference game which is a discrete version of the game defined in [3]. Let $f_{0}<\cdots<f_{K}$ be an increasing sequence of frequencies. Let $I_{k}$ be the closed interval given by $I_{k}=\left[f_{k-1}, f_{k}\right]$. We now define the approximate Gaussian interference game denoted by $G I_{\left\{I_{1}, \ldots, I_{K}\right\}}$.

Let the players $1, \ldots, N$ operate over $K$ parallel channels. Assume that the $K$ channels have coupling functions $h_{i j}(k)$. Assume that user $i$ is allowed to transmit a total power of $P_{i}$. Each player can transmit a power vector $\mathbf{p}_{n}=\left(p_{n}(1), \ldots, p_{n}(K)\right) \in\left[0, P_{n}\right]^{K}$ such that $p_{n}(k)$ is the power transmitted in the interval $I_{k}$. Therefore we have $\sum_{k=1}^{K} p_{n}(k)=P_{n}$. The equality follows from the fact that in a non-cooperative scenario all users will use all the available power. This implies that the set of power distributions for all users is a closed convex subset of the hypercube $\prod_{n=1}^{N}\left[0, P_{n}\right]^{K}$ given by:

$$
\mathbf{B}=\prod_{n=1}^{N} \mathbf{B}_{n}
$$

where $\mathbf{B}_{n}$ is the set of admissible power distributions for player $n$ given by:

$$
\mathbf{B}_{n}=\left[0, P_{n}\right]^{K} \cap\left\{(p(1), \ldots, p(K)): \sum_{k=1}^{K} p(k)=P_{n}\right\}
$$

Each player chooses a PSD $\mathbf{p}_{n}=\left\langle p_{n}(k): 1 \leq k \leq N\right\rangle \in \mathbf{B}_{n}$. Let the payoff for user $i$ be given by:

$$
C_{n}\left(\mathbf{p}_{1}, \ldots, \mathbf{p}_{N}\right)=\sum_{k=1}^{K} \log _{2}\left(1+\frac{\left|h_{n}(k)\right|^{2} p_{n}(k)}{\sum\left|h_{n m}(k)\right|^{2} p_{m}(k)+\sigma_{n}^{2}(k)}\right)
$$

where $C_{n}$ is the capacity available to player $n$ given power distributions $\mathbf{p}_{1}, \ldots, \mathbf{p}_{N}$, channel responses $h_{n}(f)$, crosstalk coupling functions $h_{m n}(k)$ and $\sigma_{n}^{2}(k)>0$ is external noise present at the $i$ 'th channel receiver at frequency $k$. In cases where $\sigma_{n}^{2}(k)=0$ capacities might become infinite using FDM strategies, however this is non-physical situation due to the receiver noise that is always present, even if small. Each $C_{n}$ is continuous in all variables.

Definition 4.1: The Gaussian Interference game $G I_{\left\{I_{1}, \ldots, I_{k}\right\}}=\{N, \mathbf{B}, \mathbf{C}\}$ is the N players non-cooperative game with payoff vector $\mathbf{C}=\left(C_{1}, \ldots, C_{N}\right)$ where $C_{n}$ are defined in (15) and $\mathbf{B}$ is the strategy set defined by (13).

The interference game is a convex non-cooperative $\mathrm{N}$-persons game, since each $\mathbf{B}_{i}$ is compact and convex and each $C_{n}\left(\mathbf{p}_{1}, \ldots, \mathbf{p}_{N}\right)$ is continuous and convex in $\mathbf{p}_{n}$ for any value of $\left\{\mathbf{p}_{m}, m \neq n\right\}$. Therefore it always has a Nash equilibrium in pure strategies. A presentation of the proof in this case using waterfilling interpretation is given in [38].

A much harder problem is the uniqueness of Nash equilibrium points in the water-filling game. This is very important to the stability of the waterfilling strategies. A first result in this direction has been given in [39]. A more general analysis of the convergence has been given in [3], [40], [41], [42] and [43]. Even the uniqueness of the Nash equilibrium, does not imply a stable dynamics. Scutari et al. [4] provided conditions for convergence. Basically, they use the Banach fixed point theorem, and require that 
the waterfilling response will be a contractive mapping. The waterfilling process has several versions: The sequential IWF is performed by a single player at each step. The parallel IWF is performed simultaneously by all players at each step, and the asynchronous IWF is performed in an arbitrary order. For good discussion of the convergence of these techniques see [4]. It should be emphasized that some conditions on the interference channel matrices are indeed required. A simple condition is strong diagonal dominance [3], and other papers relaxed these assumptions. In all typical DSL scenarios, the IWF algorithms converge. However, the convergence conditions are not always met, even in very simple cases, as the following examples shows:

Example IV - Divergence of the parallel IWF. We consider a Gaussian interference game with 2 tones and 3 players. Each player has total power $P$. The signal received by each receiver is just $y_{n}(k)=\sum_{m=1}^{3} x_{m}(k)+z_{n}(k)$ where $z_{n}(k) N\left(0, \sigma^{2}(k)\right)$, where the noise in the second band is stronger satisfying $\sigma^{2}(2)=P+\sigma^{2}(1)$. We assume simultaneous waterfilling is performed (Similar examples can be constructed for the sequential IWF algorithm, but they are omitted due to space limitations). At the first stage, all users put all their power into frequency 1 , by the first inequality. At the second stage all users see noise and interference power of $2 P+\sigma^{2}(1)$ at the first frequency, while the interference at the second frequency is $\sigma^{2}(2)=P+\sigma^{2}(1)$. Since even when all power is put into frequency 2 the total power + noise is below the noise level at frequency 1 all users will move their total power to frequency 2 . This will continue, with all users alternating between frequenies simultaneously. The average rate obtained by the simultaneous iterative waterfilling is

$$
\frac{1}{4} \log \left(1+\frac{P}{2 P+\sigma^{2}(1)}\right)+\frac{1}{4} \log \left(1+\frac{P}{3 P+\sigma^{2}(1)}\right)
$$

Nash equilibrium exists in this case, for example, two users use frequency 1 and one user uses frequency 2 resulting in a rate

$$
\frac{1}{2} \log \left(1+\frac{P}{P+\sigma^{2}(1)}\right)
$$

for each user.

The previous example demonstrated a simple condition where one of the water-filling schemes diverges. However, there are multiple NE points. The situation can be even worse. The following channel is a frequency selective channel, with a single NE in the Gaussian interference game. However non of the water filling schemes converges.

Example $V$ - Divergence of all waterfilling approaches. We provide now a second example, where both the sequential and the parallel IWF diverge, even though there is a unique NE point. Assume that we have two channels where the channel matrices $\mathbf{H}(k), k=0,1$ are equal and given by:

$$
\mathbf{H}(k)=\left[\begin{array}{lll}
1 & 0 & 2 \\
2 & 1 & 0 \\
0 & 2 & 1
\end{array}\right]
$$

and the noise at the first tone is $\sigma^{2}$ and at the second tone $\sigma^{2}+P$. Each user has total power $P$. This situation might occur when there is a strong interferer at tone 2 while the receivers and transmitters are 


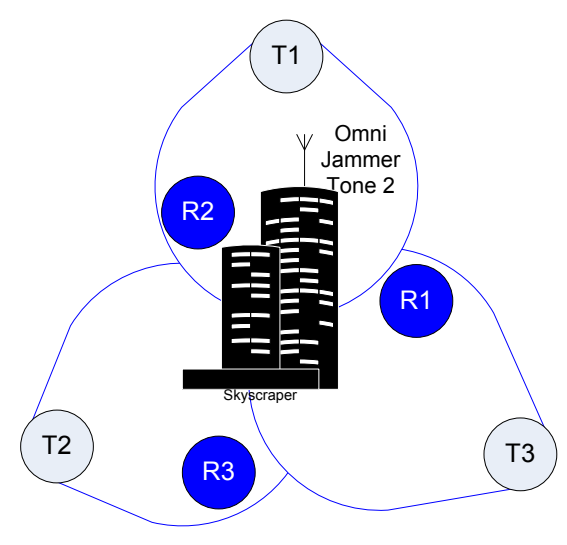

(a) Channel where the IWF fails to converge

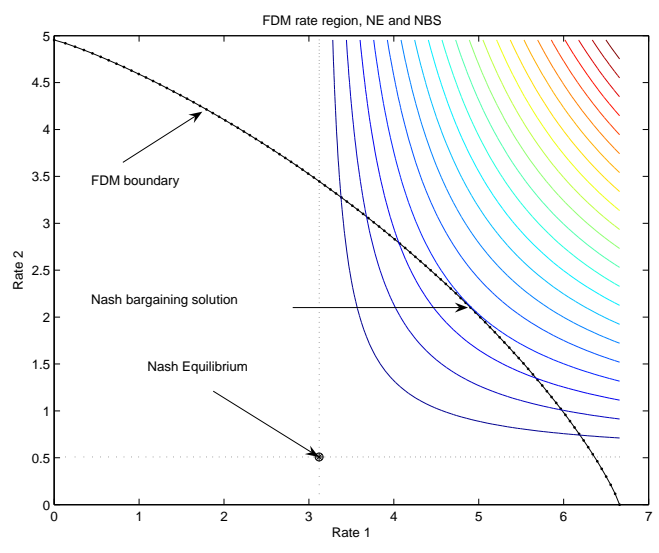

(b) Game theoretic rate region

Fig. 3. (a) A frequency selective channel with unique NE, where both sequential and parallel IWF diverge. (b)The Game Theoretic Rate region is defined by the set of rates which are better than the competitive equilibrium. The boundary of the region is exactly the set of Pareto-optimal points. The Hyperbola tangent to the rate region defines the Nash bargaining solution.

located on the sides of a triangle, each user transmitting to a receiver near the next transmitter as in figure 3(a) When the first user allocates its power it puts all its power at the first frequency. The second user chooses tone 2 . The third player puts all its power at frequency 1 , but this generates interference to user 1 which migrates to frequency 2 and we obtain that the users change their transmission tone at each step. In the simultaneous IWF all users will choose channel 1 and then migrate together to channel 2 and back. It is interesting that this game has a unique equilibrium, where each user allocates two thirds of the power to frequency 1 and one third of the power to frequency 2. Still all iterative schemes diverge.

\section{B. Pricing mechanisms for regulating distributed solutions}

To overcome some of the problems of the competitive behavior, regulation can play an important role. A generalization of the RA-IWF algorithm is the band preference (BPSM) algorithm [44] in which each user solves the following problem in parallel (or sequentially) to the other users:

$$
\begin{array}{ll}
\max _{p_{n}(1), \ldots, p_{n}(K)} & \sum_{k=1}^{K} c_{n}(k) \log _{2}\left(1+\frac{\left|h_{n n}(k)\right|^{2} p_{n}(k)}{\sum_{m \neq n}\left|h_{n m}(k)\right|^{2} p_{m}(k)+\sigma_{n}^{2}(k)}\right) \\
\text { Subject to } & P_{n}=\sum_{k=1}^{K} p_{n}(k) .
\end{array}
$$

In the BPSM algorithm the total rate is replaced by a weighted sum of the rates at each frequency. The weights can be provided by the regulator to limit the use of certain bands by strong users, so that users that suffers severe interference but do not affect other users will be protected. This results in waterfilling against a compensated noise level.

Alternative approach to the BPSM is using generalized Nash games. The basic approach has been proposed in [3] and termed Fixed Margin IWF. Each user has both power constraint, a target noise margin and a desired rate. The user minimizes its power as long as it achieves its target rate. This is a 
generalized Nash game, first analyzed by Pang et al. [5], who provided the first conditions for convergence. Noam and Leshem [6] proposed a generalization of the FM-IWF termed Iterative Power Pricing (IPP). In their solution, a weighted power is minimized, where frequency bands which have higher capacity are "reserved" to players with longer lines, through a line dependent pricing mechanism. The users iteratively optimize their power allocation so as their rate and total power constraint are maintained while minimizing the total weighted power. It can be shown that the conditions of Pang et al. can also be used to analyze the IPP algorithm. For both the BPSM and the IPP approaches, simple pricing schemes that are adapted to the DSL scenario have been proposed. The general question of finding good pricing schemes is still open, but would require to combine physical modeling of the channels as well as insight into the utilities as function of the desired rate. Even the autonomous spectrum balancing algorithm (ASB) [45] can be viewed as a generalized Nash game, where the utility is given by the rate of a fictitious reference line, and the strategy sets should satisfy both rate and power constraint. The drawback of the ASB approach here, is finding a reference line which serves as a good utility function.

\section{Bargaining over frequency selective channels under mask constraint}

In this section we define the cooperative game corresponding to the joint FDM/TDM achievable rate region for the frequency selective $N$ - user interference channel. For simplicity of presentation we limit the derivation to the two player case under PSD mask constraint. In [19] Nash bargaining solution was used for resource allocation in OFDMA systems. The goal was to maximize the overall system rate, under constraints on each users minimal rate requirement and total transmitted power. However, in that paper the solution was used only as a measure of fairness. Therefore, the point of disagreement was not taken as the Nash equilibrium for the competitive game, but an arbitrary $R_{\min }$ was used. This can result in a non-feasible problem, and the proposed algorithm might become unstable. An alternative approach is based on PSD mask constraint [21] in conjunction with general bargaining theory originally developed by Nash ([9],[37]). Based on the solution for the PSD limited case, computing the Nash Bargaining solution under total power constraint can then be solved efficiently as well [22]. In order to keep the discussion simple we concentrate the discussion on the two user PSD mask limited case.

In real applications, the regulator limits the PSD mask and not only the total power constraint. Let the $K$ channel matrices at frequencies $k=1, \ldots, K$ be given by $\left\langle\mathbf{H}_{k}: k=1, \ldots, K\right\rangle$. Player is allowed to transmit at maximum power $p_{n}(k)$ in the $k$ 'th frequency bin. In competitive scenario, under mask constraint, all players transmit at the maximal power they can use. Thus, player $n$ choose the PSD, $\mathbf{p}_{n}=\left\langle p_{n}(k): 1 \leq k \leq K\right\rangle$. The payoff for user $n$ in the non-cooperative game is therefore given by:

$$
R_{n C}(\mathbf{p})=\sum_{k=1}^{K} \log _{2}\left(1+\frac{\left|h_{n n}(k)\right|^{2} p_{n}(k)}{\sum_{m \neq n}\left|h_{n m}(k)\right|^{2} p_{m}(k)+\sigma_{n}^{2}(k)}\right) .
$$

Here, $R_{n C}$ is the capacity available to player $n$ given a PSD mask constraint distributions p. $\sigma_{n}^{2}(k)>0$ is the noise presents at the $n$ 'th receiver at frequency $k$. Note that without loss of generality, and in order to simplify notation, we assume that the width of each bin is normalized to 1 . We now define the cooperative game $G_{T F}(2, K, \mathbf{p})$. 
Definition 4.2: The FDM/TDM game $G_{T F}(2, K, \mathbf{p})$ is a game between 2 players transmitting over $K$ frequency bins under common PSD mask constraint. Each player has full knowledge of the channel matrices $\mathbf{H}_{k}$ and the following conditions hold:

1) Player $n$ transmits using a PSD limited by $\left\langle p_{n}(k): k=1, \ldots, K\right\rangle$.

2) The players are using coordinated FDM/TDM strategies. The strategy for player 1 is a vector $\boldsymbol{\alpha}_{1}=\left[\alpha_{1}(1), \ldots, \alpha_{1}(K)\right]^{T}$ where $\alpha_{1}(k)$ is the proportion of time player 1 uses the $k$ 'th frequency channel. Similarly, the strategy for player 2 is a vector $\boldsymbol{\alpha}_{2}=\left[\alpha_{2}(1), \ldots, \alpha_{2}(K)\right]^{T}$.

3) The utility of the players is given by

$$
R_{n}\left(\boldsymbol{\alpha}_{n}\right)=\sum_{k=1}^{K} \alpha_{n}(k) R_{n}(k)=\sum_{k=1}^{K} \alpha_{n}(k) \log _{2}\left(1+\frac{\left|h_{11}(k)\right|^{2} p_{n}(k)}{\sigma_{n}^{2}(k)}\right) .
$$

By Pareto optimality of the Nash Bargaining solution for each $k, \alpha_{2}(k)=1-\alpha_{1}(k)$, so we will only refer to $\boldsymbol{\alpha}=\boldsymbol{\alpha}_{1}$ as the strategy for both players. Note, that interference is avoided by time sharing at each frequency band, i.e only one player transmits with maximal power at a given frequency bin at any time. The allocation of the spectrum using the vector $\boldsymbol{\alpha}$ induces a simple convex optimization problem that can be posed as follows

$$
\begin{gathered}
\max \left(R_{1}(\boldsymbol{\alpha})-R_{1 C}\right)\left(R_{2}(\boldsymbol{\alpha})-R_{2 C}\right) \\
\text { subject to: } 0 \leq \alpha(k) \leq 1 \quad \forall k, R_{n C} \leq R_{n}(\boldsymbol{\alpha}) \quad \forall n .
\end{gathered}
$$

since the log of the Nash function (20) is a convex function the overall problem is convex. Hence, it can be solved efficiently using KKT conditions [21]. Assuming that a feasible solution exists it follows from the KKT conditions that the allocation is done according to the following rules:

1) The two players are sharing the frequency bin $k,(0<\alpha(k)<1)$ if

$$
\frac{R_{1}(k)}{R_{1}(\boldsymbol{\alpha})-R_{1 C}}=\frac{R_{2}(k)}{R_{2}(\boldsymbol{\alpha})-R_{2 C}} .
$$

2) Only player $n$ is using the frequency bin $k,\left(\alpha_{n}(k)=1\right)$, if

$$
\frac{R_{n}(k)}{R_{n}(\boldsymbol{\alpha})-R_{n C}}>\frac{R_{3-n}(k)}{R_{3-n}(\boldsymbol{\alpha})-R_{3-n, C}} .
$$

These rules can be further simplified. Let $L_{k}=R_{1}(k) / R_{2}(k)$ be the ratio between the rates at each frequency bin. We can sort the frequency bins in decreasing order according to $L_{k}$. From now on we assume that when $k_{1}<k_{2}$ then $L_{k_{1}}>L_{k_{2}}$. If all the values of $L_{k}$ are distinct then there is at most a single frequency bin that has to be shared between the two players. Since only one bin can satisfy equation (21), let us denote this frequency bin as $k_{s}$, then all the frequency bins $1 \leq k<k_{s}$ will only be used by player 1 , while all the frequency bins $k_{s}<k \leq K$ will be used by player 2 . The frequency bin $k_{s}$ has to be shared according to the rules.

We now have to find the frequency bin that has to be shared between the players if there is a solution. Let us define the surplus of players 1 and 2 when using Nash bargaining solution as $A=$ $\sum_{m=1}^{K} \alpha(m) R_{1}(m)-R_{1 C}$, and $B=\sum_{m=1}^{K}(1-\alpha(m)) R_{2}(m)-R_{2 C}$, respectively. Note that the 
ratio, $\Gamma=A / B$ is independent of frequency and is set by the optimal assignment. A-priori $\Gamma$ is unknown and may not exists. We are now ready to define the optimal assignment of the $\alpha$ 's.

Let $\Gamma_{k}$ be a moving threshold defined by $\Gamma_{k}=A_{k} / B_{k}$. where

$$
A_{k}=\sum_{m=1}^{k} R_{1}(m)-R_{1 C}, B_{k}=\sum_{m=k+1}^{K} R_{2}(m)-R_{2 C} .
$$

$A_{k}$ is a monotonically increasing sequence, while $B_{k}$ is monotonically decreasing. Hence, $\Gamma_{k}$ is also monotonically increasing. $A_{k}$ is the surplus of player 1 when frequencies $1, \ldots, k$ are allocated to player 1. Similarly $B_{k}$ is the surplus of player 2 when frequencies $k+1, \ldots, K$ are allocated to player 2 .

Let $k_{\min }=\min _{k}\left\{k: A_{k} \geq 0\right\}$, and $k_{\max }=\min _{k}\left\{k: B_{k}<0\right\}$.

Since we are interested in feasible NBS, we must have positive surplus for both users. Therefore, using the allocation rules, we obtain $k_{\min } \leq k_{\max }$ and $L_{k_{\min }} \leq \Gamma \leq L_{k_{\max }}$. The sequence $\left\{\Gamma_{m}: k_{\min } \leq m \leq\right.$ $\left.k_{\max }-1\right\}$ is strictly increasing, and always positive. While the threshold $\Gamma$ is unknown, one can use the sequences $\Gamma_{k}$ and $L(k)$ to find the correct $\Gamma$.

If there is a Nash bargaining solution, let $k_{s}$ be the frequency bin that is shared by the players. Then, $k_{\min } \leq k_{s} \leq k_{\max }$. Since, both players must have a positive gain in the game $\left(A>A_{k_{\min }-1}, B>B_{k_{\max }}\right)$. Let $k_{s}$ be the smallest integer such that $L\left(k_{s}\right)<\Gamma_{k_{s}}$, if such $k_{s}$ exists. Otherwise let $k_{s}=k_{\max }$.

Lemma 4.1: The following two statements provide the solution

1 If a Nash bargaining solution exists for $k_{\min } \leq k_{s}<k_{\max }$, then $\alpha\left(k_{s}\right)$ is given by $\alpha\left(k_{s}\right)=$ $\max \{0, g\}$, where

$$
g=1+\frac{B_{k_{s}}}{2 R_{2}\left(k_{s}\right)}\left(1-\frac{\Gamma_{k_{s}}}{L\left(k_{s}\right)}\right) .
$$

2 If a Nash bargaining solution exists and there is no such $k_{s}$, then $k_{s}=k_{\max }$ and $\alpha\left(k_{s}\right)=g$.

Based on the pervious lemmas the algorithm is described in table III] In the first stage, the algorithm computes $L(k)$ and sorts them in a non increasing order. Then $k_{\min }, k_{\max }, A_{k}$, and $B_{k}$ are computed. In the second stage the algorithm computes $k_{s}$ and $\boldsymbol{\alpha}$.

To demonstrate the algorithm we compute the Nash bargaining for the following example:

Example V: Consider two players communicating over 2x2 memoryless Gaussian interference channel with 6 frequency bins. The players' rates if they are not cooperate is $R_{1 C}=15$, and $R_{2 C}=10$. The feasible rates $R_{1}(k)$ and $R_{2}(k)$ in each frequency bin with no interference are given in Table IV after sorting the frequency bins with respect to $L_{k}$.

We now have to compute the surplus $A_{k}$ and $B_{k}$ for each players. If NBS exists then the players must have postive surplus, thus, $k_{\min }=2$, and $k_{\max }=4$. Since, $k=4$ is the first bin such that $\Gamma_{k}>L_{k}$, we can conclude that $k_{s}=4$, and $\alpha=0.33$ (using lemma 4.1). Thus, players 1 is using frequency bins 1,2 , and 3 , and using $1 / 3$ of the time frequency bin 4 . The total rate of players 1 and 2 are $40 \frac{1}{3}$ and 48 respectively.

We can also give a geometrical interpretation to the solution. In Figure 4(a) we draw the feasible total rate that player 1 can obtain as a function of the total rate of player 2 . The enclosed area in blue, is the achievable utilities set. Since, the frequency bins are sorted according to $L_{k}$ the set is convex. The 
TABLE III

ALGORITHM FOR COMPUTING THE 2X2 FREQUENCY SELECTIVE NBS

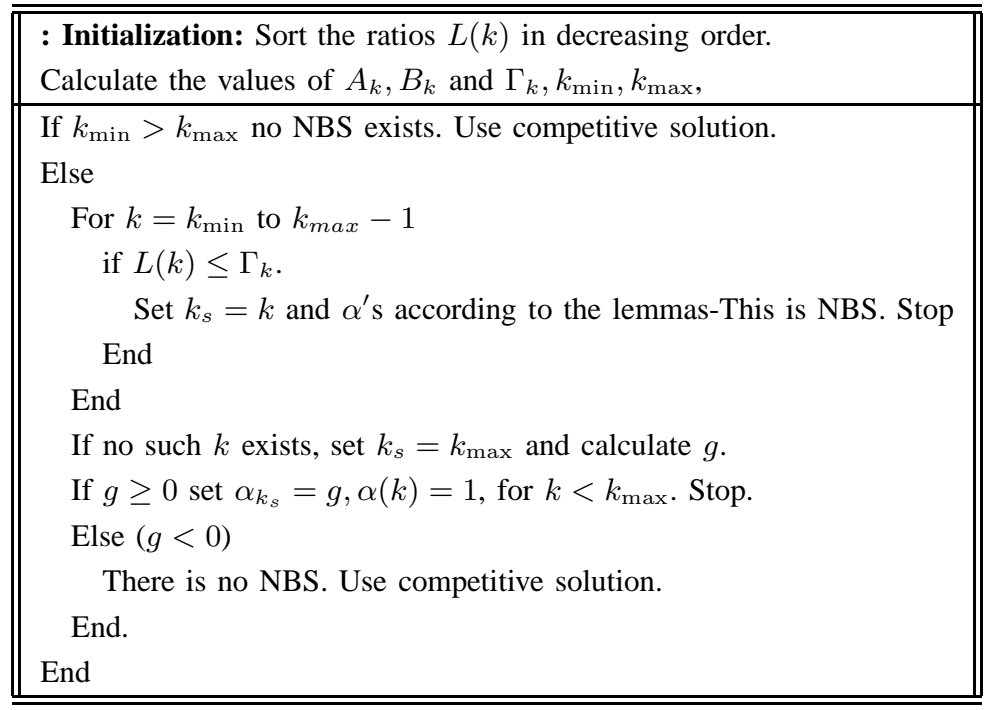

\begin{tabular}{|c|c|c|c|c|c|c|}
\hline$k$ & 1 & 2 & 3 & 4 & 5 & 6 \\
\hline$R_{1}$ & 14 & 18 & 5 & 10 & 9 & 3 \\
\hline$R_{2}$ & 6 & 10 & 5 & 15 & 19 & 19 \\
\hline$L(k)$ & 2.33 & 1.80 & 1.00 & 0.67 & 0.47 & 0.16 \\
\hline$A_{k}$ & -1 & 17 & 22 & 32 & 41 & 44 \\
\hline$B_{k}$ & 58 & 48 & 43 & 28 & 9 & -10 \\
\hline$\Gamma_{k}$ & -0.02 & 0.35 & 0.51 & 1.14 & 4.56 & -4.40 \\
\hline
\end{tabular}

TABLE IV

THE RATES OF THE PLAYERS IN EACH FREQUENCY BIN AFTER SORTING.

point $R_{C}=\left(R_{2 C}, R_{1 C}\right)=(10,15)$ is the point of disagreement. If the point $R_{C}$ is inside the achievable utility set there is a solution. The slope of the boundaries of the achievable utilities set with respect to the $-x$ axis is $L_{k}$. The vector $R_{C}-B$ connects the point $R_{C}$ and the point $\mathrm{B}$ is with the same slope with respect the $x$ axis, this is the geometrical interpretation of (21). The area of the white rectangular is the value of Nash's product function.

The results can be generalized in several directions:

First, if the values $\{L(k): k=1, \ldots, K\}$ are not all distinct then if there is a solution one can always find allocation such that at most a single frequency has to be shared.

Second, in the general case of $N$ players the optimization problem has similar KKT conditions and can be solved using convex optimization algorithm. Moreover, the optimal solution has at most $\left(\begin{array}{c}K \\ 2\end{array}\right)$ frequencies that are shared between different players. This suggests, that the optimal FDM NBS is very 


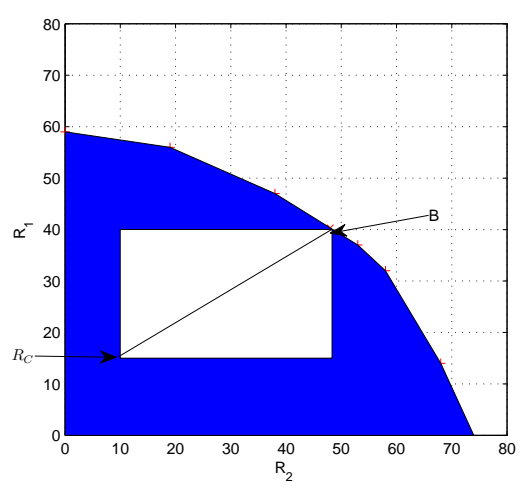

(a) The FDM/TDM rate region

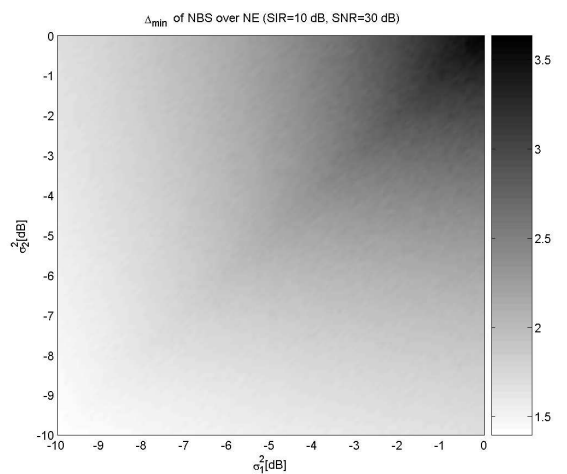

(b) Price of Anarchy for 32 parallel Rayleigh fading channels

Fig. 4. (a) Feasible FDM rate region (blue area), Nash Bargaining Solution (the area of the white rectangular) (b) Per user price of anarchy for frequency selective Rayleigh fading channel. SNR=30 dB.

close to the joint FDM/TDM solution. It is obtained by allocating the common frequencies to one of the users. Third, while the method described above fits well to stationary channels, the method is also useful when only fading statistics is known. In this case the coding strategy will change, and the achievable rate in the competitive case and the cooperative case are given by

$$
\begin{aligned}
& \tilde{\mathbf{R}}_{n C}\left(\mathbf{p}_{i}\right)=\sum_{k=1}^{K} E\left[\log _{2}\left(1+\frac{\left|h_{n n}(k)\right|^{2} p_{n}(k)}{\sum_{m \neq n}\left|h_{n m}(k)\right|^{2} p_{m}(k)+\sigma_{n}^{2}(k)}\right)\right] \\
& \tilde{R}_{n}\left(\boldsymbol{\alpha}_{n}\right)=\sum_{k=1}^{K} \alpha_{n}(k) E\left[\log _{2}\left(1+\frac{\left|h_{n n}(k)\right|^{2} p_{n}(k)}{\sigma_{n}^{2}(k)}\right)\right],
\end{aligned}
$$

respectively. All the rest of the discussion is unchanged, replacing $R_{n C}$ and $R_{n}\left(\boldsymbol{\alpha}_{n}\right)$ by $\tilde{\mathbf{R}}_{n C}, \tilde{R}_{i}\left(\boldsymbol{\alpha}_{n}\right)$ respectively. This is particularly attractive, when the computations are done in distributed way. In this case only channel state distributions are sent between the units. Hence the time scale for this data exchange are much longer. This implies that method can be used without a central control, by exchange of parameters between the units at a very low rate.

Fourth, computing the NBS under total power constraint is more difficult to solve. Several ad-hoc techniques have been proposed in the literature. Recently, it was shown that for this case there exist an algorithm which can find the optimal solution [22].

\section{SignAl PROCESSING AND COMPUTATIONAL ISSUES}

The basic requirement behind any adaptive approach for spectrum management and co-existence of communication networks, is the capability to adaptively shape the spectrum. While ten years ago this was beyond the capabilities of commercial communication systems, this is no longer the situation. The signal processing hardware available at most of the modern chips is sufficient for this purpose, and the marginal cost of this spectrum sensing and shaping capability is rapidly diminishing. The main ingredients required to implement game theoretic approaches, are varying. Typically we distinguish three 
levels of management: Autonomous, where each unit operated with no exchange of information with other units or management centers, distributed, where a management center provides some parameters to the units, which then design the spectrum and transmit by themselves, centralized where all the spectrum design is performed by a spectrum management center that collects information from units, and performs centralized processing. The competitive solutions correspond to the autonomous operation. For these, each communication unit (player) is required to have a spectral analysis module, capable of estimating channel transfer functions and noise spectrum. The regulated versions of the competitive games such as ASB, IPP and FM-IWF belong to this second family of distributed solutions where the spectrum management center provides some a-priori network information or pricing functions as well as a list of the desired rates. For the distributed solutions, a low rate communication with a center or with adjacent units is required like in the Nash bargaining solution of the previous section where only channel distributions are exchanged between users.

Finally, an important system and signal processing issue is synchronization of the various players. This is especially important for multi-carrier systems, where inter channel interference can be severe when the various units are not locked to a common time and frequency reference.

\section{ApPLICATIONS}

\section{A. Weak interference: The DSL case}

The DSL channel is an interesting example for testing algorithms emerging from game theoretic considerations. The iterative waterfilling algorithm [3] has been successfully implemented for distributed spectrum coordination of DSL lines. However the drawbacks caused by the prisoner's dilemma suggest that the strictly competitive approach (RA-IWF) is inappropriate for real life applications. Several amendments have been proposed. The first is the fixed margin iterative waterfilling [3]. In this algorithm the players are provided with a fixed target rate and each user, independently minimizes its total transmit power. As shown by Pang et al. [5] this is a generalized Nash game that converges if the interference is sufficiently weak. In [6] a generalization of the FM-IWF is proposed, that favors weak users using a pricing mechanism termed iterative power pricing. This pricing mechanism improves the performance of the FM-IWF. The game theoretic approaches have very good performance when compared to optimal spectrum management techniques, as shown in figure 5.

\section{B. Medium and strong interference - Wireless technologies}

The rapid adoption of wireless services by the public, have caused a remarkable increase in demand for reliable high data rate Internet access. This process motivated the development of new technologies. New generation of cellular systems like LTE and WiMax operating in the licensed band will be lunched in the near future. In the unlicensed band, $802.11 \mathrm{~N}$ with MIMO technology are going to become part of our daily life. The capacity of future wireless data networks will inevitably be interference limited due, the the limited radio spectrum. It is clear that any cooperation between the different networks or base stations sharing the same spectral resource can offer significant improvement in the utilization 


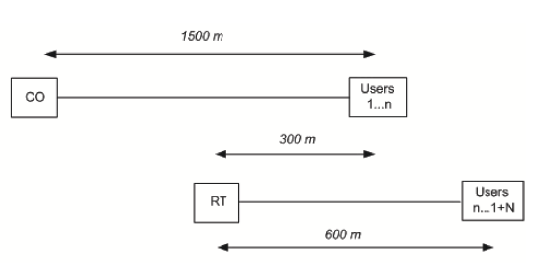

(a) Simulation setup

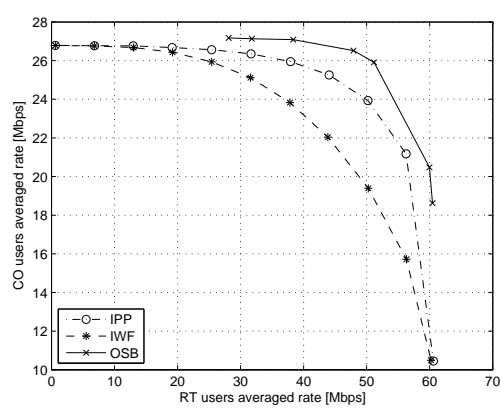

(b) Rate region

Fig. 5. (a) Simulation setup. 3 users at each location. (b) Rate regions of FM IWF, IPP and OSB. The optimal OSB is centralized and computationally very expensive. It is given as a reference to the optimal performance.

of the radio resources. Even in the same cell, cooperation between sectors can improve the over all spectral efficiency (bit/Hz/sec./sector). OFDMA technology is capable to allocate efficiently frequency bins based on the channel response of the user. In [46], a noncooperative game approach was employed for distributed sub-channel assignment, adaptive modulation, and power control for multi-cell OFDM networks. The goal was to minimize the overall transmitted power under maximal power and per user minimal rate constraints. Based on simulation results, the proposed distributed algorithm reduces the overall transmitted power in comparison with pure water-filling scheme for a seven-cell case. Kwon and Lee [47] presented a distributed resource allocation algorithm for multi-cell OFDMA systems relying on a noncooperative game in which each base station tries to maximize the system performance while minimizing the cochannel interference. They proved that there exists a Nash equilibrium point for the noncooperative game and the equilibrium is unique in some constrained environment. However, Nash equilibrium achieved by the distributed algorithm may not be as efficient as the resource allocation obtained through centralized optimization. To demonstrate the advantage of the Nash bargaining solution over competitive approaches for a frequency selective interference channel we assume that two users are sharing a frequency selective Rayleigh fading channel. The direct channels have unit fading variance and SNR of $30 \mathrm{~dB}$. The users suffer from cross interference. The cross channels fading variance was varied from $-10 \mathrm{~dB}$ to $0 \mathrm{~dB}\left(\sigma_{h_{i j}}^{2}=0.1, \ldots 1\right)$. The spectrum consisted of 32 parallel frequency bins with independent fading matrices. At each interference level of interference $\sigma_{1}^{2}=\sigma_{h_{21}}^{2}, \sigma_{2}^{2}=\sigma_{h_{12}}^{2}$ we randomly picked 25 channels (each comprising of $322 \times 2$ random matrices). The results of the minimal relative improvement (26) are depicted in figure 4(b)

$$
\Delta_{\min }=\min \left\{R_{1}^{N B S} / R_{1}^{C}, R_{2}^{N B S} / R_{2}^{C}\right\} .
$$

The relative gain of the Nash bargaining solution over the competitive solution is 1.5 to 3.5 times, which clearly demonstrates the merits of the method. 


\section{REFERENCES}

[1] E. van der Meulen, "Some reflections on the interference channel," in Communications and Cryptography: Two Sides of One Tapestry (R. Blahut, D. J. Costell, and T. Mittelholzer, eds.), pp. 409-421, Kluwer, 1994.

[2] T. Han and K. Kobayashi, "A new achievable rate region for the interference channel," IEEE Trans. on Information Theory, vol. 27, pp. 49-60, Jan. 1981.

[3] W. Yu, G. Ginis, and J. Cioffi, "Distributed multiuser power control for digital subscriber lines," IEEE Journal on Selected areas in Communications, vol. 20, pp. 1105-1115, June 2002.

[4] G. Scutari, D. Palomar, and S. Barbarossa, "Asynchronous iterative water-filling for Gaussian frequency-selective interference channels," IEEE Transactions on Information Theory, vol. 54, pp. 2868-2878, July 2008.

[5] J.-S. Pang, G. Scutari, F. Facchinei, and C. Wang, "Distributed power allocation with rate constraints in Gaussian parallel interference channels," IEEE Transactions on Information Theory, vol. 54, pp. 3471-3489, Aug. 2008.

[6] Y. Noam and A. Leshem, "Iterative power pricing for distributed spectral coordination in DSL networks," To appear in IEEE Trans. on Communications, 2009.

[7] C. Papadimitriou, "Algorithms, games and the internet," in Proc. of 34'th ACM symposium on theory of computing, pp. 749-753, 2001.

[8] A. Laufer and A. Leshem, "Distributed coordination of spectrum and the prisoner's dilemma," in Proc. of the First IEEE International Symposium on New Frontiers in Dynamic Spectrum Access Networks - DySPAN 2005, pp. 94 - $100,2005$.

[9] J. Nash, "The bargaining problem,” Econometrica, vol. 18, pp. 155-162, Apr. 1950.

[10] A. Leshem and E. Zehavi, "Bargaining over the interference channel," in Proc. IEEE ISIT, pp. 2225-2229, July 2006.

[11] H. Boche, M. Schubert, N. Vucic, and S. Naik, "Non-Symmetric Nash Bargaining Solution for Resource Allocation in Wireless Networks and Connection to Interference Calculus," in Proc. 15th European Signal Processing Conference (EUSIPCO-2007), (Poznan, Poland), Sept. 2007.

[12] E. Jorswieck and E. Larsson, "The MISO interference channel from a game-theoretic perspective: A combination of selfishness and altruism achieves pareto optimality," Acoustics, Speech and Signal Processing, 2008. ICASSP 2008. IEEE International Conference on, pp. 5364-5367, 31 2008-April 42008.

[13] J. Gao, S. A. Vorobyov, and H. Jiang, "Game theoretic solutions for precoding strategies over the interference channel," Global Telecommunications Conference, 2008. IEEE GLOBECOM 2008. IEEE, pp. 1-5, 30 2008-Dec. 42008.

[14] M. Nokleby, A. Swindlehurst, Y. Rong, and Y. Hua, "Cooperative power scheduling for wireless MIMO networks," Global Telecommunications Conference, 2007. GLOBECOM '07. IEEE, pp. 2982-2986, Nov. 2007.

[15] M. Schubert and H. Boche, "Nash bargaining and proportional fairness for log-convex utility sets," Acoustics, Speech and Signal Processing, 2008. ICASSP 2008. IEEE International Conference on, pp. 3157-3160, 31 2008-April 42008.

[16] H. Park and M. V. D. Schaar, "Bargaining strategies for networked multimedia resource management," IEEE Transactions on Signal Processing, vol. 55, pp. 3496-3511, July 2007.

[17] E. Kalai, "Solutions to the bargaining problem," Discussion Papers 556, Northwestern University, Center for Mathematical Studies in Economics and Management Science, Mar. 1983.

[18] S. Mathur, L. Sankaranarayanan, and N. Mandayam, "Coalitional games in gaussian interference channels," in Proc. IEEE ISIT, pp. 2210 - 2214, July 2006.

[19] Z. Han, Z. Ji, and K. Liu, "Fair multiuser channel allocation for OFDMA networks using the Nash bargaining solutions and coalitions," IEEE Trans. on Communications, vol. 53, pp. 1366-1376, Aug. 2005.

[20] Y. Zhao, L. kang Zeng, G. Xie, Y. an Liu, and F. Xiong, "Fairness based resource allocation for uplink OFDMA systems," The Journal of China Universities of Posts and Telecommunications, vol. 15, no. 2, pp. 50 - 55, 2008.

[21] A. Leshem and E. Zehavi, "Cooperative game theory and the Gaussian interference channel," IEEE Journal on Selected Areas in Communications, vol. 26, pp. 1078-1088, Sept. 2008.

[22] E. Zehavi and A. Leshem, "Bargaining over the interference channel with total power constraints," in Proc. of GameNets 2009, (Istanbul), May 2009.

[23] T. Cover and J. Thomas, Elements of information theory. Wiley series in telecommunications, Wiley, 1991.

[24] A. B. Carleial, "Interference channels," IEEE Transactions on Information Theory, vol. 24, pp. 60-70, Jan. 1978. 
[25] H. Sato, "The capacity of the Gaussian interference channel under strong interference," IEEE Trans. on Information Theory, vol. 27, pp. 786-788, Nov. 1981.

[26] G. Owen, Game theory. Academic Press, third ed., 1995.

[27] T. Basar and G. Olsder, Dynamic non-cooperative game theory. Academic Press, 1982.

[28] M. Osborn, Introduction to game theory. Oxford University Press, 1995.

[29] V. Gajic and B. Rimoldi, "Game theoretic considerations for the Gaussian multiple access channel," in ISIT'08 - IEEE International Symposium on Information Theory.

[30] L. Lai and H. El-Gamal, "The water-filling game in fading multiple-access channels," IEEE Transactions on Information Theory, vol. 54, no. 5, pp. 2110-2122, 2008.

[31] W. Yu, W. Rhee, S. Boyd, and J. Cioffi, "Iterative waterfilling for Gaussian vector multiple-access channels," IEEE Transactions on Information Theory, vol. 50, no. 1, pp. 145-152, 2004.

[32] W. Poundstone, Prisoner's Dilemma: John Von Neumann, Game Theory and the Puzzle of the Bomb. New York, NY, USA: Doubleday, 1992.

[33] H. Nikaido and K. Isoida, "Note on non-cooperative convex games," Pacific Journal of Matematics, vol. 5, pp. 807-815, 1955.

[34] F. Meshkati, H. Poor, and S. Schwartz, "Energy-efficient resource allocation in wireless networks," Signal Processing Magazine, IEEE, vol. 24, pp. 58-68, May 2007.

[35] K. Arrow and G. Debreu, "The existence of an equilibrium for a competitive economy," Econometrica, vol. XXII, pp. 265290, 1954.

[36] T. Ichishi, Game theory for economic analysis. Academic Press,, 1983.

[37] J. Nash, "Two-person cooperative games," Econometrica, vol. 21, pp. 128-140, Jan. 1953.

[38] A. Laufer, A. Leshem, and H. Messer, "Game theoretic aspects of distributed spectral coordination with application to DSL networks.” arXiv:cs/0602014, 2006.

[39] S. Chung, J. Lee, S. Kim, and J. Cioffi, "On the convergence of iterative waterfilling in the frequency selective Gaussian interference channel," Preprint, 2002.

[40] S. Chung and J. Cioffi, "Rate and power control in a two-user multicarrier channel with no coordination: the optimal scheme versus a suboptimal method," IEEE Transactions on Communications, vol. 51,, pp. 1768 - 1772, Nov. 2003.

[41] Z. Luo and J. Pang, "Analysis of iterative waterfilling algorithm for multiuser power control in digital sibcriber lines," EURASIP Journal Applied Signal Processing, vol. 2006, pp. 1-10, 2006.

[42] G. Scutari, D. Palomar, and S. Barbarossa, "Asynchronous iterative water-filling for Gaussain frequency-selective interference channles: A unified framework," in IEEE SPAWC-2006, July 2006.

[43] K. W. Shum, K.-K. Leung, and C. W. Sung, "Convergence of iterative waterfilling algorithm for Gaussian interference channels," IEEE Journal on Selected Areas in Communications, vol. 25, pp. 1091-1099, August 2007.

[44] W. Lee, Y. Kim, M. H. Brady, and J. M. Cioffi, "Band-preference dynamic spectrum management in a DSL environment," in Proc. IEEE Globecom 2006, (San Francisco, CA), IEEE, Nov. 2006.

[45] R. Cendrillon, J. Huang, M. Chiang, and M. Moonen, "Autonomous spectrum balancing for digital subscriber lines," IEEE Trans. on Signal Processing, vol. 55, pp. 4241 - 4257, August 2007.

[46] Z. Han, Z. Ji, and K. Liu, "Power minimization for multi-cell OFDM networks using distributed non-cooperative game approach," Global Telecommunications Conference, 2004. GLOBECOM '04. IEEE, vol. 6, pp. 3742-3747 Vol.6, Nov.-3 Dec. 2004.

[47] H. Kwon and B. G. Lee, "Distributed resource allocation through noncooperative game approach in multi-cell OFDMA systems," Communications, 2006. ICC 'O6. IEEE International Conference on, vol. 9, pp. 4345-4350, June 2006. 